\title{
A bow shock flow containing (almost) all types of ('exotic') MHD discontinuities
}

\author{
H. De Sterck, H. Deconinck, S. Poedts, and D. Roose
}

\begin{abstract}
We present 2D numerical simulations of a planar field-aligned ideal Magnetohydrodynamic (MHD) bow shock flow in a regime where fast MHD switch-on shocks are possible. In this regime, the interaction of the 3 nonlinear waves of the non-strictly hyperbolic MHD system leads to a complicated solution with interacting shocks of different MHD shock type. Fast and slow shocks and tangential discontinuities are present in the flow. Moreover, we clearly identify non-classical intermediate shocks and shocks with a compound structure, which are a manifestation of the non-convex nature of the hyperbolic MHD system. We discuss some numerical problems encountered when high-resolution numerical MHD schemes derived from common Computational Fluid Dynamics approaches are used to simulate this kind of flows. This bow shock flow simulation is extremely simple in setup, but contains a wealth of interacting MHD shocks, which makes it a good new test case for ideal MHD codes. Observations suggest that the MHD shock formation effects of our simulations may occur in processes in the corona of the sun.
\end{abstract}

Accepted for the Proceedings of the Seventh International Conference on Hyperbolic Problems (ETH Zuerich, February 9-13, 1998).

\section{Introduction and setup of the numerical simulation}

The equations for the hyperbolic system of ideal one-fluid Magnetohydrodynamics (MHD) in conservative form are given by

$$
\frac{\partial}{\partial t}\left[\begin{array}{c}
\rho \\
\rho \vec{v} \\
\vec{B} \\
e
\end{array}\right]+\nabla \cdot\left[\begin{array}{c}
\rho \vec{v} \\
\rho \vec{v} \vec{v}+I(p+\vec{B} \cdot \vec{B} / 2)-\vec{B} \vec{B} \\
\vec{v} \vec{B}-\vec{B} \vec{v} \\
(e+p+\vec{B} \cdot \vec{B} / 2) \vec{v}-(\vec{v} \cdot \vec{B}) \vec{B}
\end{array}\right]=0 .
$$

Here $\rho$ and $p$ are the density and pressure of the fully ionized plasma, $\vec{v}$ is the plasma velocity, $\vec{B}$ the magnetic field, and

$$
e=\frac{p}{\gamma-1}+\rho \frac{\vec{v} \cdot \vec{v}}{2}+\frac{\vec{B} \cdot \vec{B}}{2}
$$


is the total energy density of the plasma. $I$ is the unity matrix. The magnetic permeability $\mu=1$ in our units. We take $\gamma=5 / 3$ for the adiabatic index.

The MHD conservation laws describe a hyperbolic system which allows for 3 linear wave modes, the fast, the Alfvén and the slow wave, with (positive) anisotropic wave speeds satisfying $c_{f}>c_{A}>c_{s}$ in standard notation. The wave speeds can coincide, which makes the MHD system non-strictly hyperbolic. The MHD system is non-convex $([2,5])$.

In this paper we present numerical simulation results describing the stationary bow shock formed when a uniform flow of a magnetized fluid falls in on a perfectly conducting cylinder $(r=0.125)$ in 2D. This stationary ideal MHD problem is completely specified by the value of the plasma $\beta=2 p / B^{2}$ and the fast Mach number $M_{f}=v / c_{f}$ of the incoming flow. We take $(\rho=1, p=0.2, \vec{B}=(1,0)$, $\vec{v}=(1.5,0))$ for the incoming flow. Thus $\beta=0.4$ and $M_{f}=1.5$. The flow speed is faster than the fast MHD speed, so we expect that a steady bow shock will form. The flow speed lies in the region where fast switch-on shocks can be expected (see Sec. 2). The incoming magnetic field and velocity field lie in the same plane and are parallel. It can be proven that this alignment is conserved everywhere for a steady state solution, resulting in a planar field-aligned flow in the whole domain.

In Fig. 1a we show a general view of the resulting bow shock flow. The leading shock front contains a 'dimpled' concave-outward (from the cylinder) central part. The leading shock front is followed by a second front, and additional discontinuities can be seen. In this paper we give a consistent physical interpretation for all the features present in this complicated flow.

Bow shocks are present in space physics plasmas when the solar wind encounters comets or planets. Many numerical simulations have been done to describe such bow shocks (e.g. [4]). Shock formation in the switch-on regime has been studied in the context of fast solar Coronal Mass Ejections (CMEs) $([12,13])$. However, to our knowledge, the steady bow shock in the switch-on regime has not been studied yet, and the interacting shock solution reported in this paper has not been described before. Much research is going on in the development of shock-capturing numerical schemes for MHD (e.g. [11, 4]) and attempts are being made to extend standard techniques from Computational Fluid Dynamics (CFD) ([5]) to MHD. We will discuss some aspects of how to use these techniques for flows with interacting MHD shocks.

This paper is organized as follows. In Sec. 2 we give a brief overview of the properties of MHD discontinuities, which will help us to explain why the traditional bow shock topology of Fig. 2a is not possible in the flow regime under consideration. In Sec. 3 we present a consistent physical interpretation of all the features present in the simulation results. In Sec. 4 we discuss the numerical technique used. In Sec. 5 we discuss the relevance of our simulation results for the problem of the physical existence of non-classical intermediate shocks and compound structures, which are composed of a shock with an attached rarefaction and are a manifestation of the non-convex nature of the MHD system $([2,14,8,9])$. 
We report on the possible observation of the shock formation effects described in this paper in the propagation of solar CMEs. We conclude in Sec. 6.

\section{MHD discontinuities and the symmetries of the bow shock flow}

The MHD conservation laws allow for many types of discontinuities ([6]). Fast

shocks refract $\vec{B}$ away from the normal of the shock surface. The normal plasma velocity (in the shock frame) is greater than the normal fast MHD speed upstream of the fast shock, such that the upstream fast Mach number $M_{f_{1}}=v_{x_{1}} / c_{f_{1}}$ is greater than 1. (The regions upstream and downstream from the shock are traditionally denoted 1 and 2 . The $x$ direction is normal to the shock front, and $y$ is tangential.) The flow is sub-fast downstream, so the downstream fast Mach number is smaller than 1 . Intermediate shocks change the sign of the tangential component of $\vec{B}$, and the upstream Alfvénic Mach number $M_{A_{1}}>1$, whereas downstream $M_{A_{2}}<1$. Slow shocks refract $\vec{B}$ towards the normal.

There are limit cases for these three kinds of shocks. Fast switch-on shocks have $B_{y_{1}}=0$ but $B_{y_{2}}$ does not vanish (it is 'switched on'), so the magnetic field is refracted away from the normal over a finite angle $\theta$. The downstream (normal) Alfvénic Mach number $M_{A_{2}}$ exactly equals 1. Intermediate shocks can have $B_{y_{1}}=B_{y_{2}}=0$, in which case they are called hydrodynamic (or parallel) shocks. Slow switch-off shocks switch off the tangential component of the magnetic field, and thus $B_{y_{2}}=0$, and $M_{A_{1}}=1$. Switch-on shocks will only occur for plasma speeds in the switch-on region

$$
c_{f_{1}}<v_{x_{1}}<c_{f_{1}} \sqrt{\frac{\gamma(1-\beta)+1}{\gamma-1}}
$$

where $c_{f}$ is the (normal) fast speed. Switch-on shocks can only be found in low$\beta$ plasmas. Other MHD discontinuities include contact, tangential and rotational discontinuities.

So why do we not find a classical concave-inward bow shock solution (Fig. 2a) in our simulation? The answer can be found in the analysis of the symmetry of an MHD flow in the switch-on regime (as was first done in $[12,13]$ ). If we look at the symmetry of a concave-inward bow shock solution (Fig. 2a), we see that the direction of the $\vec{B}$ field can not change on the stagnation streamline, which is a line of symmetry. When we move downward along the shock front and approach the stagnation streamline, the fast shock will approach a fast switch-on shock $\left(B_{y_{1}}=0\right)$, which will however still turn $\vec{B}$ over a finite angle $\theta$. This is a discontinuity between streamlines, and this simple bow shock topology is thus not possible. In stead, the complex topology of Fig. 2b, satisfying the symmetry constraints by the presence of intermediate shocks, is found as the result of the numerical simulation. 


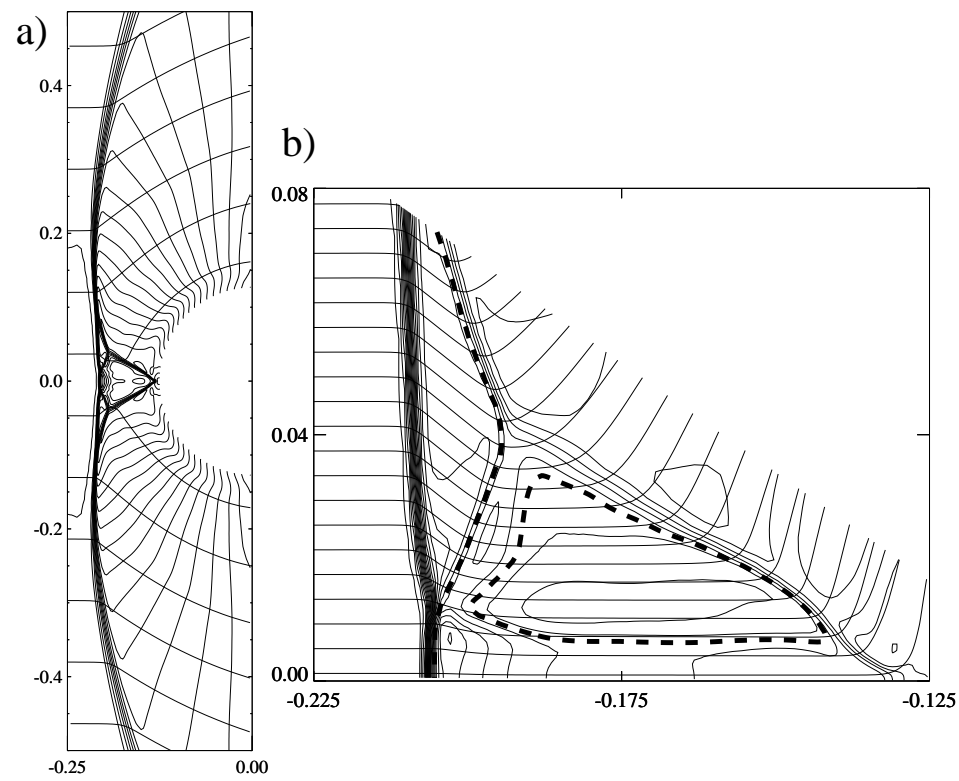

Figure 1. (a) Global view of the new bow shock solution. We show density contours and magnetic field lines. The flow comes in from the left. The cylinder fills the space of the white half disc on the right. (b) Detail of the solution upward from the symmetry axis. We show Alfvénic Mach number contours and magnetic field lines. The dashed line is a contour where the Alfvénic Mach number exactly equals 1 .

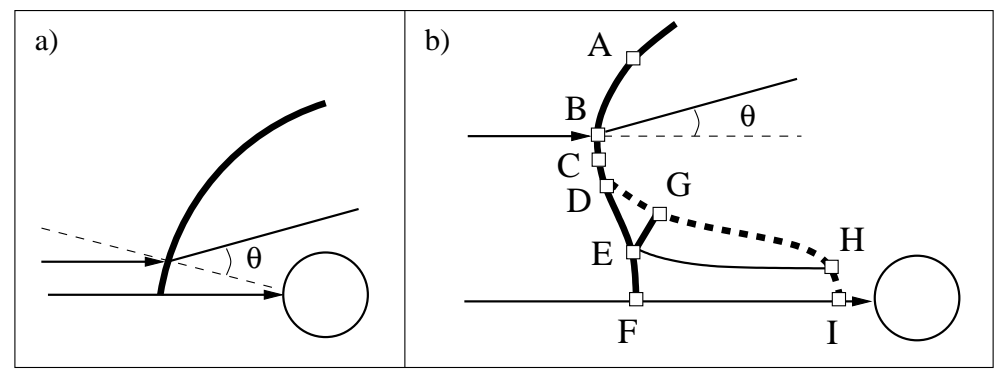

Figure 2. Possible bow shock topologies for a uniform flow (magnetic field lines have arrows) falling in from the left on a conducting cylinder. Shock normals are shown as dashed lines. (a) Traditional concave-inward (to the cylinder) geometry. (b) Interacting shock topology of our simulation results. 


\section{Interpretation of the simulation results}

In Fig. 1b we show a detail of the flow. We use the lettering of Fig. 2b to identify the shock parts. A-B and D-E are fast shocks because the field is refracted away from the normal. E-F is a hydrodynamic shock because the field lines are not refracted. E-G is an intermediate shock, because it clearly contains the $M_{A}=1$ contour (meaning that the flow goes from super-Alfvénic to sub-Alfvénic while passing through the shock), and because the field lines are flipped over the normal. E-F is very reminiscent of a Mach stem as encountered in Hydrodynamic shock reflection. D-G-H-I is a slow switch-off shock, because the upstream Alfvénic Mach number is equal to 1 , and because the downstream magnetic field is normal to the shock surface. E-H is a tangential discontinuity. Other tangential discontinuities are stretching out from points $\mathrm{G}$ and $\mathrm{H}$ along the streamlines to infinity.

In Fig. 3a-d we show plots of the variables along the stagnation streamline. Going from left to right, we see two jumps in the variables, for instance in the pressure. The first jump corresponds to the hydrodynamic shock at point $\mathrm{F}$. The Alfvénic Mach number jumps from above 1 to under 1, indicating an intermediate shock. The magnetic field does not show a jump, consistent with a purely hydrodynamic shock. Going further to the right, we see that we pass through a rarefaction. The hydrodynamic shock together with the rarefaction make up a compound structure. Then we reach a constant state, before the quantities jump again near the cylinder, where the slow switch-off shock is encountered at point I. We see that the entropy is well conserved on this streamline in the smooth parts of the flow.

In Fig. 3e-f we show a cut along a ray through the center of the cylinder cutting the leading front between points $\mathrm{C}$ and $\mathrm{D}(\theta=47.66$ degrees $)$. We cross only one shock, which brings the Alfvénic (and the fast) Mach number from above 1 to under 1, so B-C-D is clearly a non-classical intermediate shock. Looking at the magnetic field, we see a clear compound structure (a shock followed by a rarefaction) for this shock as well.

\section{Numerical method}

We have simulated the flow in the upper left quadrant, on a $120 \times 120$ stretched elliptic polar-like structured grid, extending to $x=-0.35$ on the $x$-axis, and to $y=1.4$ on the $y$-axis (see Fig. 4a for a detail of the grid). Starting from a uniform flow initial condition, the flow evolves until a steady state bow shock solution is obtained. We solve Eq. (1) using a finite volume high resolution Godunov shock capturing scheme which is second order in space and time, using a MUSCL approach ([5]) with minmod-limiting on the slopes of the primitive variables. The time-integration is explicit with a 2-step Runge-Kutta method.

We use the Lax-Friedrichs numerical flux function, which is simple and robust. Contact and tangential discontinuities are not very well resolved due to high 
dissipation for these waves, but shocks are well resolved in a steady state calculation. Roe's scheme ([11]) in theory would resolve shocks and especially the tangential discontinuities much better, but we found several problems while trying to apply this scheme to our simulation. Roe's scheme suffers from various instabilities, like the carbuncle-instability ([10]) where the shock front intersects the symmetry axis, and this makes that a steady state solution can not be obtained. Adding dissipation to the numerical scheme (for instance using the H-correction proposed in [7]) did seem to cure the carbuncle up to moderate grid resolution, but other instabilities remained. It seems to be difficult to find a good parameter free change to Roe's numerical scheme which solves the problems in a general way. Using the Lax-Friedrichs scheme however, we obtained good convergence of the density residual (Fig. 4b).

We use a projection scheme to keep the $\nabla \cdot \vec{B}=0$ constraint satisfied up to machine accuracy in the chosen discretization ([1]). As can be seen in Fig. 5, the divergence wave technique proposed by Powell ([4]) gives almost exactly the same solution. However, at the stagnation point close to the cylinder, the two approaches give a slightly different solution.

We carefully checked if the results we obtained do not contain numerical artefacts or features dependent on the numerical resistivity. We did many simulations with grids of different cell sizes and we obtained uniform grid convergence to the solution shown in Fig. 1. We also did simulations on uniform grids and grids with a different accumulation of points than in the grid of Fig. 4a, and refinement studies using these different grids resulted in grid convergence to the same solution of Fig.
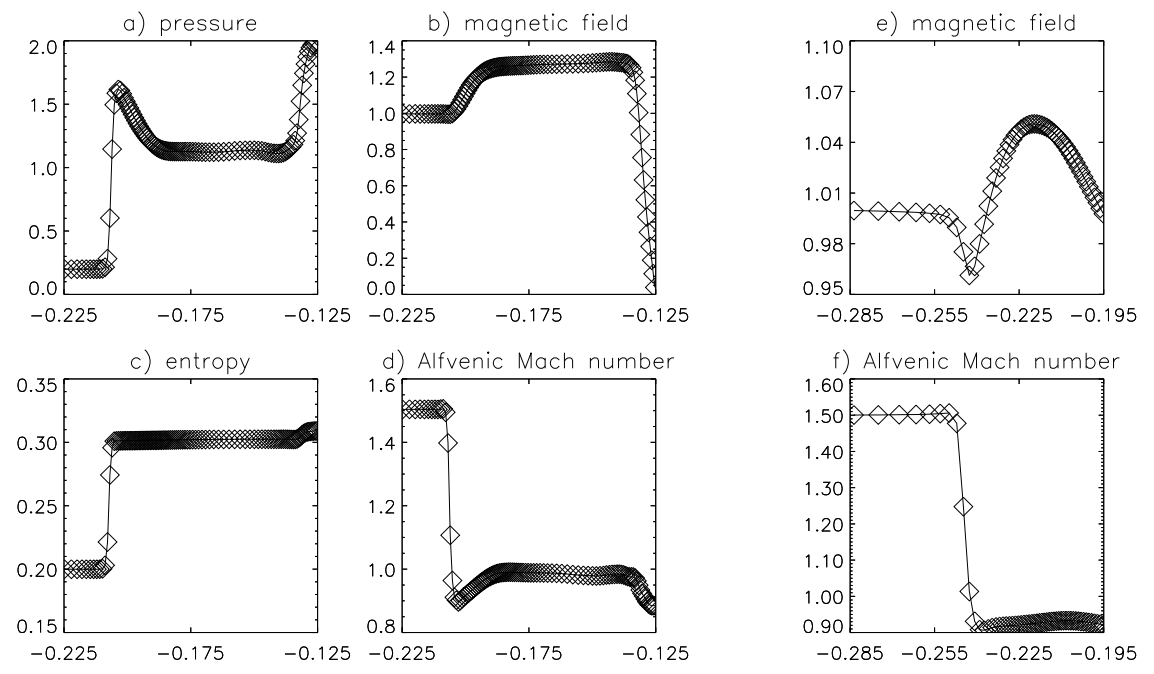

Figure 3. (a-d) Plots of some variables along the stagnation streamline. (e-f) Cut along a ray between points $\mathrm{C}$ and D. 
a)

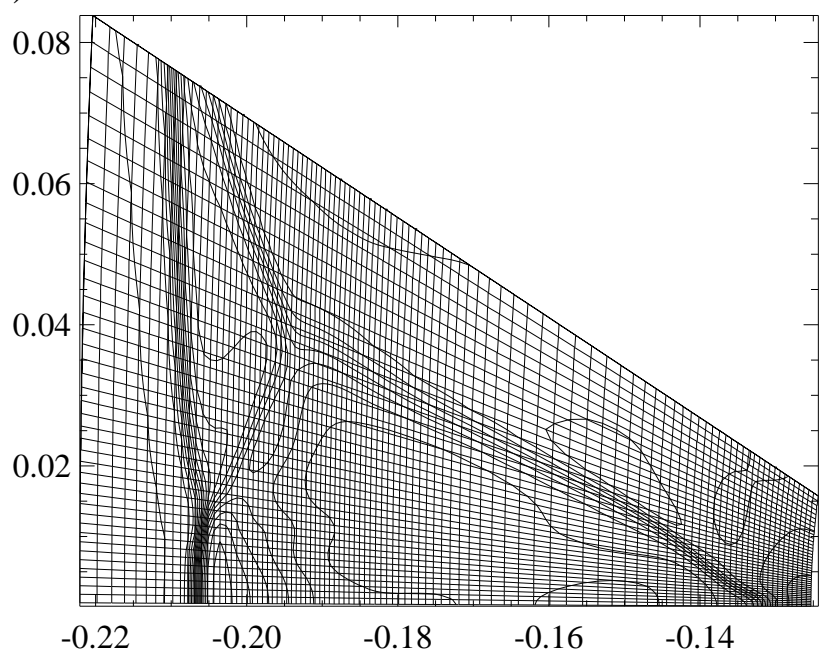

b)

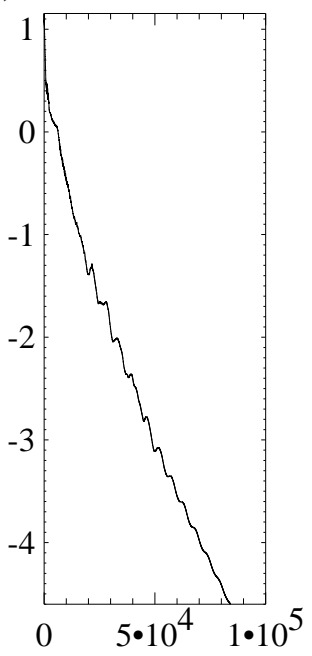

FiguRE 4. (a) Detail of the simulation grid with density contours. (b) Convergence of the simulation to a steady state. The logarithm of the root mean square of the density residual is shown as a function of the number of iterations.

1. The fully consistent and complete physical interpretation of all the features in the solution gives further confidence that the features present in our solution are real and physically meaningful.

\section{Intermediate and compound shocks and solar Coronal Mass Ejections}

Because the MHD system is not convex, compound shocks can exist in principle $([5,2])$. Intermediate shocks satisfy the MHD Rankine-Hugoniot relations. They are called non-classical shocks because more than 1 family of characteristics converges into the shock. MHD intermediate and compound shocks were commonly believed to be unphysical ([6]) or 'exotic'. Intermediate shocks and shocks with a compound structure are however clearly present in our results, and this for the first time in steady 2D ideal MHD simulations. This is a strong indication that those types of discontinuities have to exist, at least in the planar case. Our findings are consistent with earlier 1D ideal MHD simulations, where compound shocks are

found ([2]). They are also consistent with recent results on the admissibility of 


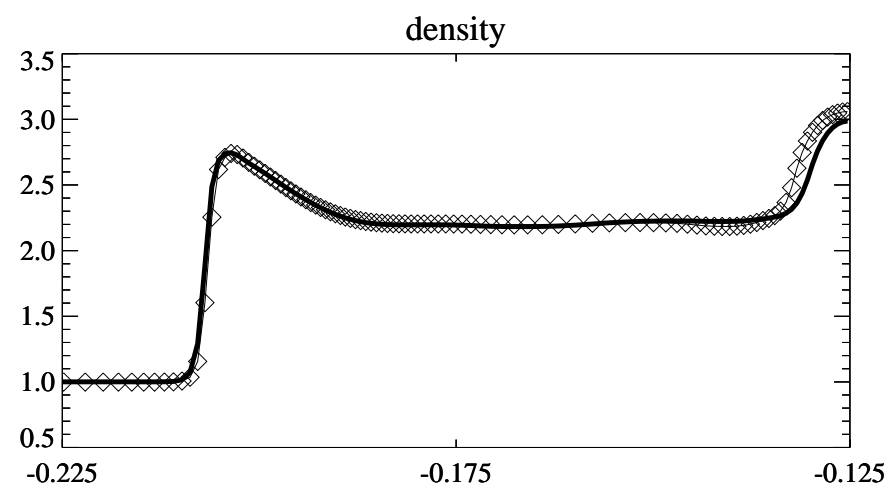

Figure 5. Comparison of the density on the stagnation streamline, using a projection scheme (thin line with diamond symbols) or Powell's source term (thick line). The solutions are almost identical, except near the stagnation point close to the cylinder (on the right).

MHD intermediate shocks $([14,8,9])$. Compound shocks were shown to be necessary and stable ingredients for the solution of some planar MHD Riemann problems $([8,9])$. It is however not clear in how far the steady compound structures of our simulations are related to the time-dependent compound shocks encountered in Riemann problems.

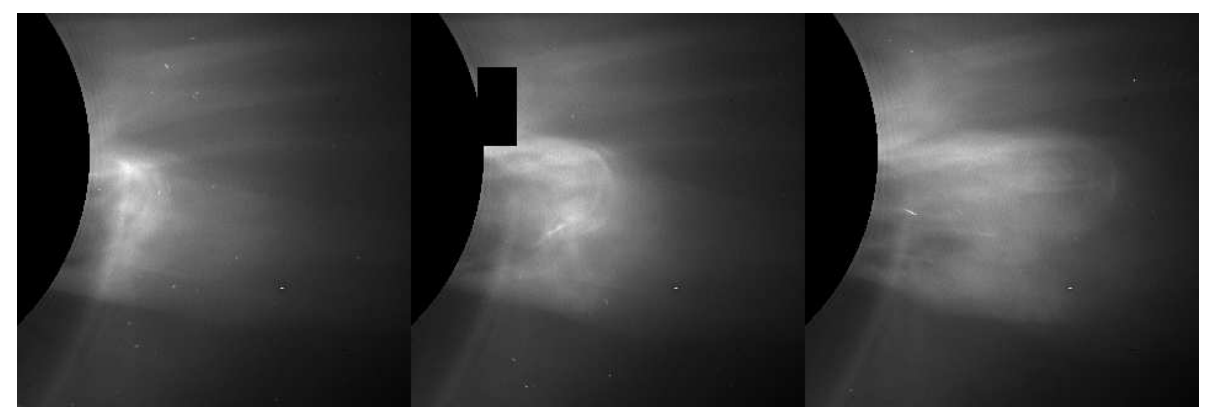

Figure 6. Observation of a solar CME with the LASCO C2 coronagraph. The black disc covers the sun and has a radius of approximately $2 \times \mathrm{R}_{\text {sun }}$. The images, taken on September 28, 1997, at $16: 12,16: 31$, and 16:53 UT (from left to right), seem to show the evolution of an initially concave-inward (to the sun) CME front to a dimpled front with a double-loop appearance. 
The shock formation effects described in this paper could occur in low- $\beta$ space plasmas. Solar CMEs are one example. Up to several times a day, large-scale structures in the solar corona disrupt and are ejected out of the corona. Some of these ejecta move away from the sun in the solar coronal plasma with super-fast nearly field-aligned speeds lying in the switch-on region, inducing shock fronts preceding the ejecta, much like the cylinder in our simulations can be thought of as to induce a shock front while moving with a constant speed into a static plasma. Some evidence for the shock formation effects seen in our simulations may be found in coronagraph images of these CMEs. As already noted in $([12,13])$ and as can be seen in Fig. 6, the leading feature of many fast CMEs, which can be interpreted as the signature of a fast shock front, seems to show a dimpled front, consistent with the dimple in the leading front of our simulation results (Fig. 1a). Moreover, the density depletion in the V-shaped region between the two shock fronts in our simulation (Fig. 1a) divides the downstream flow into two distinct lobes with higher density. This structure may explain why some CMEs seem to evolve to a double-loop appearance (Fig. 6 and [3]).

\section{Conclusion}

In this paper we have discussed simulation results for a model problem describing a planar field-aligned MHD bow shock flow. The interaction of the three types of nonlinear waves in the hyperbolic system leads to a complicated flow with a wealth of interacting discontinuities of different types. We have given a clear and consistent physical interpretation of this flow pattern. Non-classical intermediate shocks and shocks with a compound structure, which are a manifestation of the non-convex nature of the hyperbolic MHD system, are clearly identified. Our results are the first clear illustration in 2D of recent findings on the admissibility of non-classical intermediate shocks and compound shocks that were obtained in a 1D framework. High-resolution numerical MHD schemes derived from common CFD approaches are successful in describing this kind of flows, if care is taken about the $\nabla \cdot \vec{B}=0$ constraint and about the carbuncle phenomenon. Our simulation, very simple in setup, but producing a wealth of interacting discontinuities, is a good new test case for ideal MHD codes. The shock formation effects present in this model problem may occur in the propagation of fast solar CMEs and other phenomena in low- $\beta$ space plasmas.

\section{Acknowledgments}

HDS acknowledges numerous helpful discussions with B. C. Low and A. Hundhausen. The SOHO/LASCO data used here are produced by a consortium of the Naval Research Laboratory (USA), Max-Planck-Institut fuer Aeronomie (Germany)), Laboratoire d'Astronomie (France), and the University of Birmingham (UK). SOHO is a project of international cooperation between ESA and NASA. The National Center for Atmospheric Research is sponsored by the National Science Foundation (USA). HDS is a Research Assistant of the Fund for 
Scientific Research - Flanders (Belgium), and is also at the Centre for Plasma Astrophysics. SP is a Research Associate of the Fund for Scientific Research Flanders (Belgium).

\section{References}

[1] J. U. Brackbill and D. C. Barnes, J. Comput. Phys. 35, 426 (1980).

[2] M. Brio and C. C. Wu, J. Comput. Phys. 75, 400 (1988).

[3] H. De Sterck, The MHD shock separation effect and the double-loop appearance of fast CMEs, submitted to Geophys. Res. Lett. (1998).

[4] T. I. Gombosi, K. G. Powell, and D. L. De Zeeuw, J. Geophys. Res. 99, 21,525 (1994).

[5] R. J. Leveque, Numerical methods for conservation laws, Lectures in Mathematics ETH Zurich (Birkhauser Verlag, Basel, 1992).

[6] M. A. Liberman and A. L. Velikovich, Physics of shock waves in gases and plasmas, Vol. 19 of Springer Series in Electrophysics (Springer-Verlag, Berlin, 1985).

[7] E. Morano, R. Sanders, and M.-C. Druguet, Multidimensional dissipation for upwind schemes: stability and applications to gas dynamics, preprint (1997).

[8] R. S. Myong and P. L. Roe, J. Plasma Physics 58, 485 (1997).

[9] R. S. Myong and P. L. Roe, J. Plasma Physics 58, 521 (1997).

[10] J. J. Quirk, Int. J. Numer. Methods Fluids 18, 555 (1994).

[11] P. L. Roe and D. S. Balsara, SIAM J. Appl. Math. 56, 57 (1996).

[12] R. S. Steinolfson and A. J. Hundhausen, J. Geophys. Res. 95, 6389 (1990).

[13] R. S. Steinolfson and A. J. Hundhausen, J. Geophys. Res. 95, 20,693 (1990).

[14] C. C. Wu, J. Geophys. Res. 100, 5579 (1995).

High Altitude Observatory,

NCAR, Boulder, Co, USA

E-mail address: desterck@ucar.edu

von Karman Institute for Fluid Dynamics,

Brussels, Belgium

E-mail address: deconinck@vki.ac.be

Centre for Plasma Astrophysics,

K.U. Leuven, Belgium

E-mail address: stefaan.poedts@wis.kuleuven.ac.be

Department of Cumputer Science,

K.U. Leuven, Belgium

E-mail address: dirk.roose@cs.kuleuven.ac.be 